\title{
Smoothing images creates corners
}

Sporring, Jon; Olsen, Ole Fogh; Nielsen, Mads; Weickert, Joachim

Published in:

Image and Vision Computing

Publication date:

2000

Document version

Early version, also known as pre-print

Citation for published version (APA):

Sporring, J., Olsen, O. F., Nielsen, M., \& Weickert, J. (2000). Smoothing images creates corners. Image and Vision Computing, 18(3), 261-266. 


\title{
Smoothing images creates corners
}

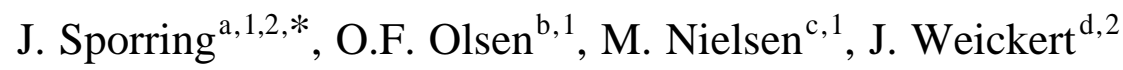 \\ ${ }^{a}$ Institute of Computer Science, Science and Technology Park of Crete, Vassilika Vouton, P.O. Box 1385, GR 711 10 Heraklion, Crete, Greece \\ ${ }^{\mathrm{b}}$ Department of Computer Science, University of Copenhagen, Universitetsparken 1, DK-2100 Copenhagen, Denmark \\ ${ }^{\mathrm{c}}$ The IT University in Copenhagen, Glentevej 67, DK-2400 Copenhagen, Denmark \\ ${ }^{\mathrm{d}}$ Computer Vision, Graphics and Pattern Recognition Group, Department of Mathematics and Computer Science, University of Mannheim, \\ D-68131 Mannheim, Germany
}

Received 26 January 1998; received in revised form 4 May 1999; accepted 25 June 1999

\begin{abstract}
The isophote curvature times the gradient magnitude to some power has been studied in the literature as a measure of cornerness in images. Stability in terms of sampling noise has been proposed by selecting corners at high scale and locating them at fine scale. We will examine the problem of tracking extrema of these measures in the Linear Scale-Space using catastrophe theory. This enables us to explain potential dangers of commonly used corner detectors. We conclude that creations and annihilations generically occur, implying that corners in general cannot be tracked to arbitrary fine (or coarse) scale. Experiments on real-world images confirm the practical relevance of our investigations. (C) 2000 Elsevier Science B.V. All rights reserved.
\end{abstract}

Keywords: Corners; Isophote curvature; Gradient magnitude; Linear scale-space; Catastrophe theory

\section{Introduction}

Corner detection plays a central role in many image analysis applications ranging from character recognition to landmark identification. The literature on corner detection roughly divides into two classes. Some use explicit models $[1,2]$, while others use derivative expressions like the Gaussian curvature, the structure tensor (interest operator, second moment matrix), expressions involving the isophote curvature, and the curvature of Canny edges [5,6].

One subclass of the latter is corners defined as the isophote curvature times the absolute gradient magnitude to some power $a$ :

$C(x, y, a)=|\nabla L(x, y)|^{a} \kappa(x, y)=L_{w}(w, v)^{a-1} L_{v v}(w, v)$,

where $L$ is an image, $|\nabla L|$ is its gradient magnitude, and $k$ is its isophote curvature image. Further, $(w, v)$ is a local right hand coordinate system, where $w$ is the gradient direction of $L$ and $v$ the (perpendicular) tangent direction of the isophote. Subscript denotes differentiation. Kitchen and Rosenfeld [8] suggested to use $a=1$, Zuniga and Haralick [9] proposed

\footnotetext{
* Corresponding author. Tel.: +30-81-391-701; fax: +30-81-391-601.

${ }^{1}$ Supported by the Danish National Research Council.

${ }^{2}$ Supported by EC contract no. ERBFMRY-CT96-0049 (VIRGO http:// www.ics.forth.gr/virgo) under the TMR programme.
}

$a=0$, and Blom [10], Lindeberg [11], and Alvarez et al. [12] investigated $a=3$.

The advantage of using a corner measure with $a>0$ is that the product will focus on high isophote curvatures close to high contrast edges. There are two special values of $a$ that deserve a note: $a=0$ is invariant under monotonic transformation of the image intensities (morphological invariance), and $a=3$ is invariant under affine transformations (the angle of the corner).

Scale is an essential part of detecting corners. At pixel scale, differential corner measures will be dominated by the pixellation, but a simple smoothing reduces the effect of the pixel grid, while still retaining important features. We will investigate the above subclass of corner measures in "linear scale-space" (see [13] and the references therein):

$L(x, y, t)=G(x, y, t) * L(x, y)$,

where the original image $L(x, y)$ is convolved with a Gaussian $G(x, y, t)=(1 /(4 \pi t)) \exp \left(-\left(x^{2}+y^{2}\right) /(4 t)\right)$ of variance $2 t$. The parameter $t$ is called scale. Henceforth, we will call "linear scale-space" for scale-space. There exist essentially three implementations of scale-space: Spatial convolution, multiplication in the frequency domain, and direct solution of the heat equation $L_{t}=L_{x x}+L_{y y}$. We use here the frequency implementation, since it is fastest for large scales, and it easily extends to the calculation of derivatives. 

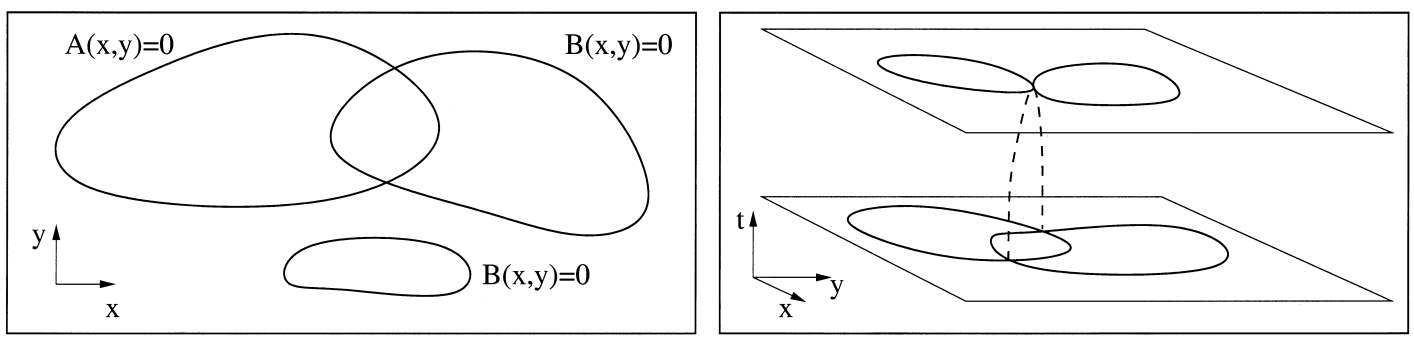

Fig. 1. The superficial and deep structure of the intersection of curves. Left: two curves $A$ and $B$ intersect generically in an even number of points or not at all. Right: a stack of images illustrating the evolution of an intersection (dashed line). Top image corresponds to an annihilation event. A creation event is identical with reversed scale.

However, we note that for small scales the direct solution of the heat equation is more stable [14].

The advantage of such an embedding is that it reduces the grid and noise effects and allows for a uniform analysis of corners of all sizes or resolutions. The disadvantage is that the corners are dislocated at high scale and should be tracked back to low scale in order to improve their location. Such an algorithm typically involves the following steps:

1. produce a stack of images $L(x, y, t)$ for a range of scales;

2. for each scale and some constant, $a \in\{0,1,2,3\}$, calculate the corner image $C(x, y, a, t)$;

3 . detect corner points in each corner image;

4. link corner points across scales;

5. at fine scale, choose the corner points that can be linked to coarser scale.

An essential part of the algorithm is thus the linking of corners across scales. We will show that this process - although common in the literature-is problematic, since new corners may arise as the scale is increased.

Studying the behaviour of corners across scales is conveniently done with catastrophe theory. We will sketch the catastrophe structure in two different settings. Firstly, we will examine the unconstrained, spatial singularity structure of $C(x, y, a, t)$. We find that new corners do generically appear for increasing scales. Secondly, we extend Rieger's analysis [15] to single isophotes, since the definition of $C(x, y, a, t)$ is intrinsically linked to isophotes, and since the corners do lie on the curves under study in contrast to Canny edges as noted by Rieger [15]. Also for single isophotes, creations of corners do occur for increasing scales.

Some authors have studied corners by first obtaining edge curves, and then analyse the curves for corners, see e.g. Ref. [5] and references therein. These studies do typically not analyse the effect of image resolution.

Related to our work in terms of catastrophe theory is Damon [16], Rieger [15,17], Griffin and Colchester [18], Olsen [19], Johansen [20], and Gauch and Pizer [21].

\section{Image structure}

Corners are spatial point features, and for the differential corner measures (1), these points are found as the intersection between curves: $A(x, y)=0$ and $B(x, y)=0$. We will investigate two pairs of intersecting curves:

1. corners defined as critical points of $C$, i.e. $A=C_{x}$ and $B=C_{y}$;

2. corners defined on the isophote, $L_{0}$, as extrema $C$ along the isophote, i.e. $A=L-L_{0}$ and $B=C_{v}$.

We analyse the solution set satisfying $A=B=0$ and its evolution, when $L$ is evolved in scale-space.

In Fig. 1 (left) are shown typical intersections between curves: either two curves intersect with non-parallel tangents or their intersection is empty. This typical type of intersection is called transverse. A consequence of the transverse intersection is that closed curves given by zero-crossings of any pair of image derivatives have an even number of intersections or none at all.

The intersection points form smooth curves in scalespace. Such feature curves will have points, where the tangent is perpendicular to the $t$-axis. This typically corresponds to the interaction of spatial point features, i.e. feature points annihilate or are created in pairs as illustrated in Fig. 1 (right). We refer to such points as catastrophe points.

It is always possible to come up with an example where the intersection set exhibits extreme and atypical behaviour. However, in this paper we will only consider typical behaviour (in a well defined mathematical manner). We will consider properties which hold for an open ${ }^{3}$ and dense ${ }^{4}$ subset of all images. Such properties are called generic, and corresponds well with the general notion of being typical: an image picked at random will with probability 1 be generic, and any image is infinitesimally close to a generic image [18]. We will now illustrate how transverse intersections in jet space describe geometric properties of our feature points, where after we will discuss the genericity of transverse intersections.

\footnotetext{
${ }^{3}$ A set $X$ is open if $\forall x \in X \exists \epsilon>0: N_{\epsilon}(x) \subset X$, where $N_{\epsilon}(x)$ is an $\epsilon$ neighbourhood of $x$.

${ }^{4} \mathrm{~A}$ set $X$ is dense in $Y$ if and only if $\forall y \in Y \forall \epsilon>0: N_{\epsilon}(y) \cap X \neq \varnothing$.
} 

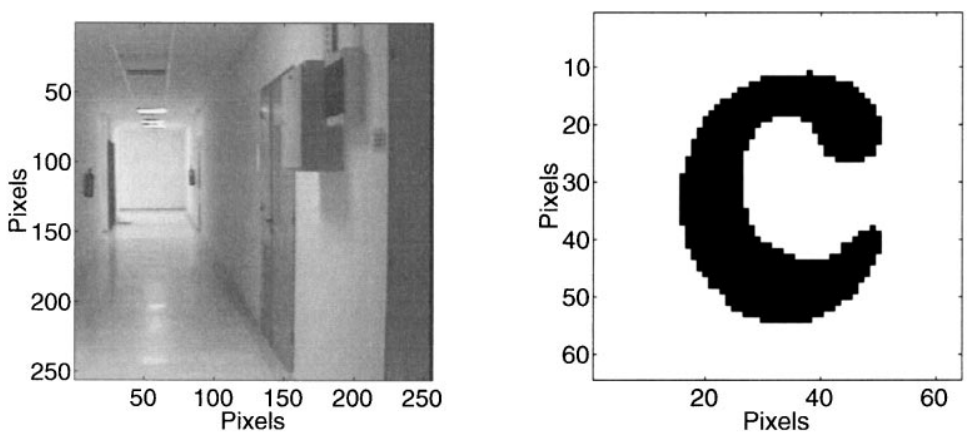

Fig. 2. Two test images. Left: a typical hallway, courtesy C. Colios, Foundation of Research and Technology—Hellas. Right: The letter 'c'.

\subsection{Geometric properties by transverse intersections in jet space}

We shall establish generic properties in jet space (the space of $k$-th order Taylor expansions of solutions to the heat equation, $\left.L_{t}=L_{x x}+L_{y y}\right)$. A property is represented by its defining equations $(A=B=0)$ expressed in terms of image derivatives. This implicitly defines a manifold of some co-dimension ${ }^{5}$ called the property manifold. To establish generic properties, we have to show that the property manifold is defined and determine its co-dimension.

A scale-space image can be mapped to a smooth manifold in jet space in the following way: The $k$-th order local Taylor expansion is in each point of the scale-space mapped into jet space, thus sweeping a manifold, which we call the image manifold.

If the image manifold is transverse to the property manifold, then the set of points in scale-space, where the property occurs, is a smooth manifold with the same co-dimension as the property manifold. Hence we have described our feature manifold in scale-space.

\subsection{Transverse intersections are generic}

The scale-space images which have a transverse intersection between the image manifold and the property manifold form an open and dense set in the space of all scale-space images. This result is due to Damon [16]. Note that a scalespace image satisfies the heat equation, hence the evolution of spatial feature points is restricted in comparison to a general evolution. This changes the general program of catastrophe theory slightly [16].

By the above we conclude that typical feature properties can be determined by transverse intersection between images and a property in jet space.

\subsection{Generic catastrophes for differential corner measures}

The co-dimension of a manifold describes the number of

\footnotetext{
${ }^{5}$ The co-dimension of a manifold is the dimension of the embedding space minus the dimension of the manifold. E.g. the co-dimension of a sphere is always 1 , while its dimension depends on the embedding space: A sphere in 3D is two-dimensional and in $4 \mathrm{D}$ is three-dimensional.
}

constraints imposed on the degree of freedoms in the embedding space. We will now illustrate how the co-dimension for corner feature curves is found.

With some local parametrisation of the feature curve is an implicit parametrisation given by:

$A(x(s), y(s), t(s))=0$,
$B(x(s), y(s), t(s))=0$.

Differentiating with respect to the parameter $s$ yields:

$A_{x} x_{s}+A_{y} y_{s}+A_{t} t_{s}=0$,

$B_{x} x_{s}+B_{y} y_{s}+B_{t} t_{s}=0$.

In a catastrophe point $\left(t_{s}=0\right)$ and with a none-zero curve tangent we have:

$A_{x} B_{y}-B_{x} A_{y}=0$.

We have analysed (2) combined with $A=B=0$, and we have determined that they define a smooth manifold of codimension 3 in jet space. The equations $A=B=0$ alone define a property manifold of co-dimension 2 . The solutions to $A=B=0$ therefore are smooth curves in scale-space with catastrophes occurring in isolated points on these curves. Geometrically, catastrophes for critical points in $C$ correspond to the annihilation or creation of a saddle with either a minimum or a maximum. The catastrophes for the isophote constrained measure correspond to the annihilation or creation of a minimum and maximum pair.

The sign of $t_{s s}$ determines the catastrophe type: negative is an annihilation and positive is a creation. We have been able to create generic examples of both types, hence both annihilation and creation can occur. If $t_{s s}=0$, we get a catastrophe of even higher order. Most cases, where $t_{s}=t_{s s}=0$, define a manifold of co-dimension 4 and are not generic, since scale-space images only have 3 dimensions. The exception is the case $a=3$, where higher order events happen generically, where $C=C_{x}=C_{y}=0$. These points are not corners however.

We conclude that tracking a corner over scale can only be performed for an open interval of scales. At scales outside this interval, the corner does not exist. Hence, both annihilations and creations are generic events. In 

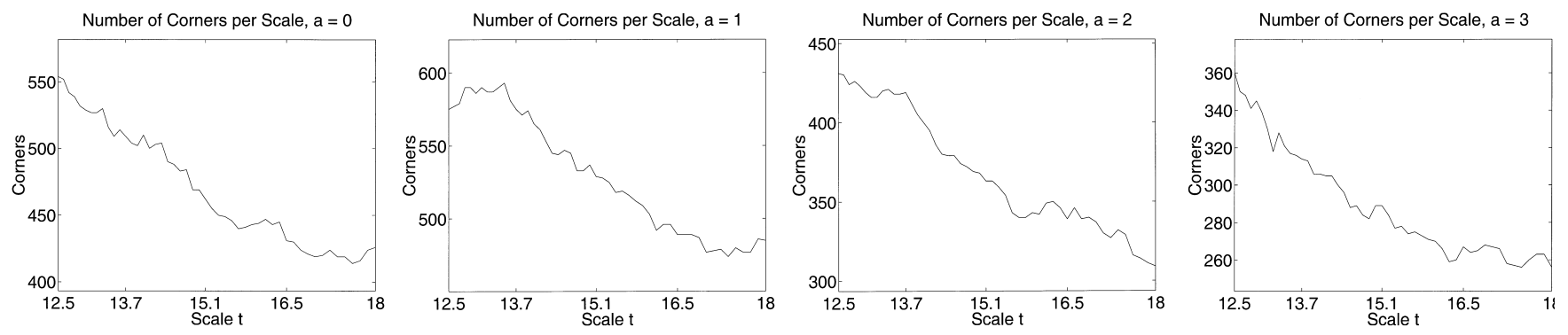

Fig. 3. Corners of Fig. 2 (left) given by $C_{x}=C_{y}=0$ have frequent creations for all $a=0, \ldots, 3$.

particular, it is in general not possible to track corners to arbitrary fine scales.

\section{Experiments on two typical images}

The above analysis shows that in the continuous case, annihilations and especially creations are likely when scale is increased. To verify these results in the discrete setting we have calculated corners of two typical images shown in Fig. 2. The number of corners as a function of scale for Fig. 2 (left) is shown in Fig. 3. The corners are found as points where $|C|$ is larger than its 4 neighbours. We see that it is not a monotonic decreasing sequence and conclude that creations do occur. In Fig. 4 are shown a sequence of scales for $a=0$ in which a corner creation occurs approximately in the middle image. The creation events for other values of $a$ are similar. We thus conclude that corners defined as $C_{x}=C_{y}=0$ also have creations in the discrete setting.

We have tested the isophote approach $\left(L=L_{0}\right.$ and $C_{v}=0$ ) on the image in Fig. 2 (right). For binary images the mid-isophote is an edge operator, and it is to be expected that the mid-isophote is close to $L_{w w}=0$ edges for low scales. However, binary images are not generic. Fortunately, an arbitrary small scale larger than zero will transform a binary image into a generic gray-valued image.

The number of corners for a range of scales for the mid- isophote is shown in Fig. 5. Corners are here found as points in which $|C|$ is larger than its 2 neighbours on the isophote. Again we see that this is not a monotonic decreasing sequence and conclude that the isophote approach also has creations in the discrete setting.

The final experiment we have performed concerns the selection of semantically important corners: Some approaches order the extrema of $C$ according to their absolute strength at coarse scales and uses this ordering to choose the semantically important extrema at fine scale [11]. Varying $a$ can alter the ordering dramatically and possibly catastrophically. In Fig. 6 we show the value of $C$ on the mid-isophote of the letter 'c' at scale 12.5 for various $a$ values. Notice the sharp peak for $a=0$ at arclength 40. This corresponds to the sharpest turn of the contour of the letter ' $c$ '. For $a=2$ this peak is practically removed and replaced by the neighbouring peak at arclength 20. This shift in ordering also occurs for corners given by $C_{x}=C_{y}=0$.

\section{Summary}

We have studied the family of corner measures $\kappa|\nabla L|^{a}$ for $a \in\{0, \ldots, 3\}$ when the image is embedded in "linear scalespace". Two approaches have been analysed: the catastrophe structure of the spatial extrema of the corner measure and the extrema along an isophote.
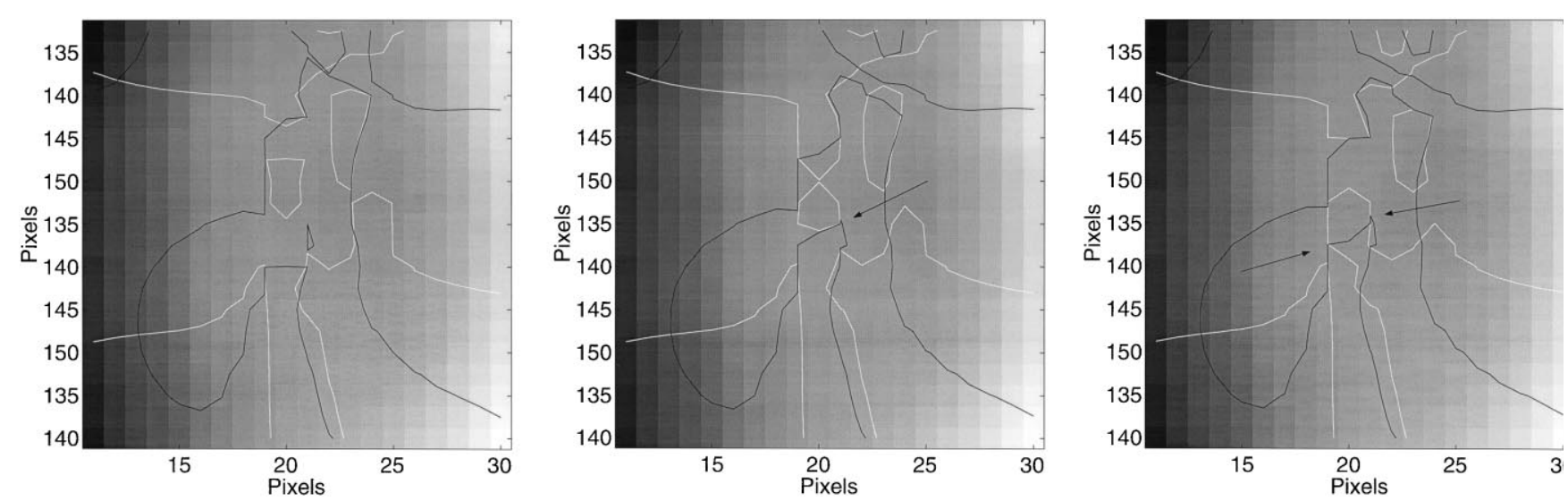

Fig. 4. A zoom of Fig. 2 (left) showing lines of $C_{x}=0$ (light) and $C_{y}=0$ (dark) for $a=0$ and scale $t=17.60,17.70,17.86$ from left to right. Arrows mark creations. 

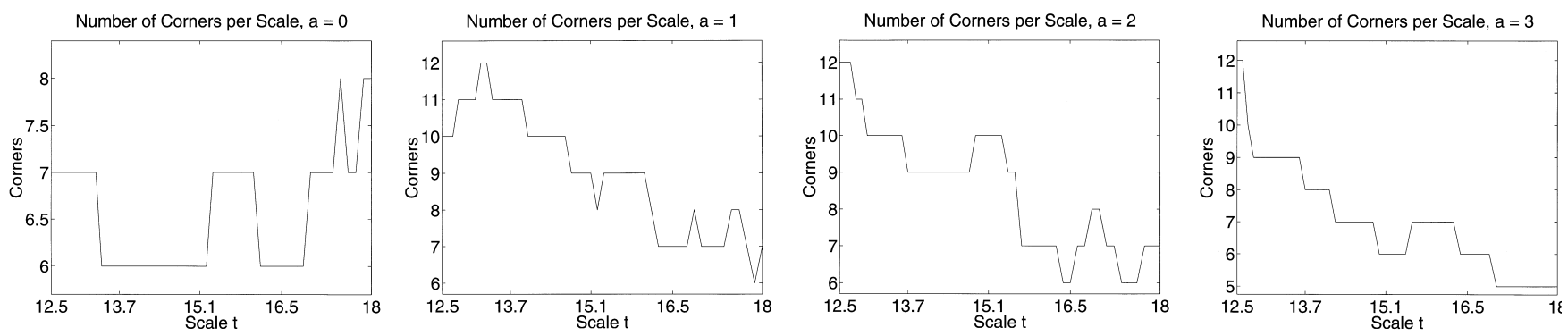

Fig. 5. Corners given by $L=L_{0}$ and $C_{v}=0$ have frequent creations for all $a=0, \ldots, 3$.
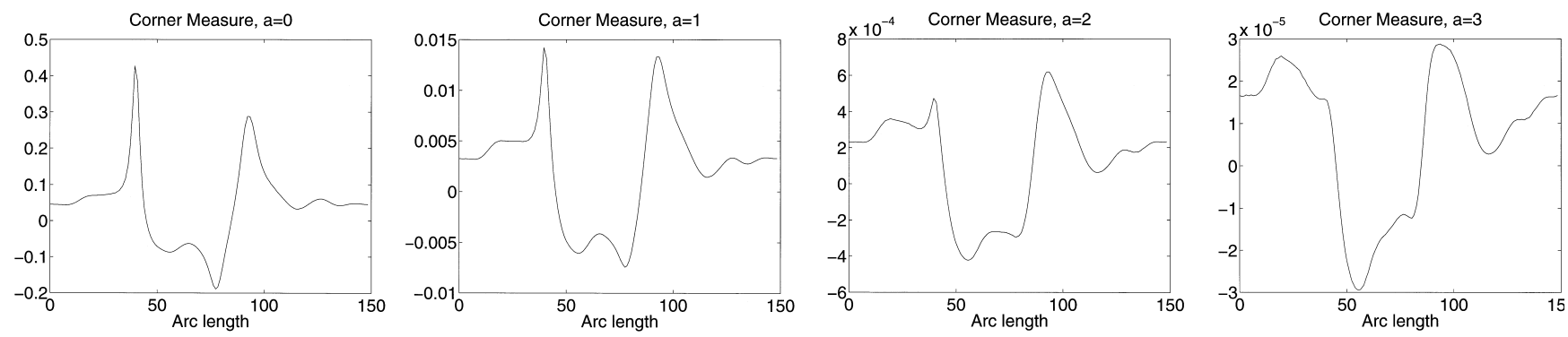

Fig. 6. The function $C$ on the mid-isophote for Fig. 2 (right) at $t=12.5$ and for all $a=0, \ldots, 3$. The arc-length functions starts on the outside of 'c' at its leftmost point and travels downwards.

We have found in both approaches that the evolution of corner points are smooth curves in space and scale, and that both annihilations and creations are generic events at isolated points on these curves. Hence, corners cannot always be tracked to arbitrary coarse or fine scale.

For both approaches we have experienced that the value $a=1$ seems to generate the fewest number of creations. Coincidentally, this is also the measure with the simplest intrinsic form: $C(x, 1, t)=L_{v v}$. Conversely, we have experienced that especially many catastrophes occur when $a=0$.

Finally, the isophote approach has been shown to shift the focus away from high isophote curvature points for $a>0$. The resulting corners do not always correspond well with intuition. A similar result holds for corners defined as spatial extrema of $C$.

\section{References}

[1] V. Berzins, Accuracy of Laplacian edge detectors, Computer Vision, Graphics, and Image Processing 27 (1984) 195-210.

[2] K. Rangarajan, M. Shah, D. Van Brackle, Optimal corner detector, Computer Vision, Graphics, and Image Processing 48 (1989) 230245.

[3] K. Rohr, Modelling and identification of characteristic intensity variations, Image and Vision Computing 10 (2) (1992) 66-76.

[4] M.A. Shah, R. Jain, Detecting time-varying corners, Computer Vision, Graphics, and Image Processing 28 (3) (1984) 345-355.

[5] F. Mokhtarian, R. Suomela, Robust image corner detection through curvature scale space, IEEE Transactions on Pattern Analysis and Machine Intelligence 20 (12) (1998) 1376-1381.

[6] H.-H. Nagel, Displacement vectors derived from second-order intensity variations in image sequences, Computer Vision, Graphics, and Image Processing 21 (1983) 85-117.

[7] K. Rohr, Localization properties of direct corner detectors, Journal of Mathematical Imaging and Vision 4 (1994) 139-150.

[8] L. Kitchen, A. Rosenfeld, Gray-level corner detection, Pattern Recognition Letters 1 (1982) 95-102.

[9] O.A. Zuniga, R. Haralick, Corner detection using the facet model, in Proc. of the IEEE Conf. on Computer Vision and Pattern Recognition, 1983, pp. 30- 37.

[10] J. Blom, Topological and geometrical aspects of image structure, $\mathrm{PhD}$ thesis, Department of Medical and Physiological Physics, University of Utrecht, The Netherlands, 1992.

[11] T. Lindeberg, Scale-Space Theory in Computer Vision, Kluwer, Boston, 1994

[12] L. Alvarez, F. Morales, Affine morphological multiscale analysis of corners and multiple junctions, International Journal of Computer Vision 25 (1997) 95-107.

[13] J. Weickert, S. Ishikawa, A. Imiya, On the history of Gaussian scalespace axiomatics, in: J. Sporring, M. Nielsen, L. Florack, P. Johansen (Eds.), Gaussian Scale-Space Theory, Kluwer, Dordrecht, 1997, pp. 45-59.

[14] J. Weickert, Anisotropic Diffusion in Image Processing, Teubner Verlag, Stuttgart, 1998.

[15] J. Rieger, Generic properties of edges and corners on smooth greyvalue surfaces, Biological Cybernetics 66 (1992) 497-502.

[16] J. Damon, Local Morse theory for Gaussian blurred functions, in: J. Sporring, M. Nielsen, L. Florack, P. Johansen (Eds.), Gaussian ScaleSpace Theory, Kluwer, Dordrecht, 1997, pp. 147-163.

[17] J. Rieger, Generic evolution of edges on diffused greyvalue surfaces, Journal of Mathematical Imaging and Vision 5 (1995) 207-217. 
[18] L.D. Griffin, A.C.F. Colchester, Superficial and deep structure in linear diffusion scale space: Isophotes, critical points and separatrices, Image and Vision Computing 13 (7) (1995) 543-557.

[19] O.F. Olsen, Multi-scale watershed segmentation, in: J. Sporring, M. Nielsen, L. Florack, P. Johansen (Eds.), Gaussian Scale-Space Theory, Kluwer, Dordrecht, 1997, pp. 191-200.
[20] P. Johansen, Local analysis of image scale space, in: J. Sporring, M. Nielsen, L. Florack, P. Johansen (Eds.), Gaussian Scale-Space Theory, 1997, pp. 139-148.

[21] J.M. Gauch, S.M. Pizer, Multiresolution analysis of ridges and valleys in grey-scale images, IEEE Transactions on Pattern Analysis and Machine Intelligence 15 (6) (1993) 635-646. 